\title{
Semi-Supervised Learning of Hidden Markov Models via a Homotopy Method
}

\author{
Shihao Ji, Layne T. Watson, Fellow, IEEE, and Lawrence Carin, Fellow, IEEE
}

\begin{abstract}
Hidden Markov model (HMM) classifier design is considered for analysis of sequential data, incorporating both labeled and unlabeled data for training; the balance between labeled and unlabeled data is controlled by an allocation parameter $\lambda \in[0,1)$, where $\lambda=0$ corresponds to purely supervised HMM learning (based only on the labeled data) and $\lambda=1$ corresponds to unsupervised HMM-based clustering (based only on the unlabeled data). The associated estimation problem can typically be reduced to solving a set of fixed point equations in the form of a "natural-parameter homotopy". This paper applies a homotopy method to track a continuous path of solutions, starting from a local supervised solution $(\lambda=0)$ to a local unsupervised solution $(\lambda=1)$. The homotopy method is guaranteed to track with probability one from $\lambda=0$ to $\lambda=1$ if the $\lambda=0$ solution is unique; this condition is not satisfied for the HMM, since the maximum likelihood supervised solution $(\lambda=0)$ is characterized by many local optimal solutions. A modified form of the homotopy map for HMMs assures a track from $\lambda=0$ to $\lambda=1$. Following this track leads to a formulation for selecting $\lambda \in[0,1)$ for a semi-supervised solution, and it also provides a tool for selection from among multiple (local optimal) supervised solutions. The results of applying the proposed method to measured and synthetic sequential data verify its robustness and feasibility compared to the conventional EM approach for semi-supervised HMM training.
\end{abstract}

\section{Index Terms}

Hidden Markov models (HMMs), homotopy method, supervised learning, semi-supervised learning.

S. Ji and L. Carin are with the Department of Electrical and Computer Engineering, Duke University, Box 90291, Durham, NC 27708-0291; L. T. Watson is with the Departments of Computer Science and Mathematics, Virginia Polytechnic Institute and State University, Blacksburg, VA 24061-0106. 


\section{INTRODUCTION}

A classifier is typically trained on data pairs, defined by feature vectors and corresponding class labels. Such a framework is called supervised learning. In most cases class labels are manually assigned by experts. It is therefore often expensive and time consuming to collect large quantities of labeled data. Because of this labeling cost, labeled data are often scarce in practice. Using limited labeled data, a classifier designed with supervised training is often unreliable, manifesting poor generalization performance [1].

To overcome this problem a new technique, termed semi-supervised learning, has been proposed, in which unlabeled data (for which only the feature vectors are available) are integrated with labeled data when performing classifier design. Because unlabeled data can be collected easily, without labeling costs, semi-supervised learning has attracted interest in various applications, for data defined by single feature vectors [2]-[6] and for sequential data defined by a sequence of feature vectors [7].

This paper focuses on sequential data, modeled via hidden Markov models (HMMs) [8]. HMMs have underlying (hidden) states, with the sequence of states characterized via a Markov process. These models have been used extensively in two application areas. The first application is concerned with classification of sequential data in speech recognition [8], target classification [9], and computational biology [10], etc. In these tasks, given a sequence of data, HMMs

are used to assign a class label to the entire sequence. The second application deals with estimating the underlying Markovian state sequence given observed sequential data. Examples of this application include part-of-speech tagging in natural language processing [11] and namedentity extraction in information extraction [12]. This paper concentrates on the first application, corresponding to classification of sequential data, with an HMM classifier designed by exploiting both labeled and unlabeled data sequences. Labeled data correspond to sequences for which the corresponding target classes are known, and unlabeled data correspond to sequential data without the corresponding labels.

A conventional semi-supervised approach for training a generative model (e.g., HMMs) is the expectation maximization (EM) algorithm [3], [13]. In this procedure, the labels of the unlabeled 
data are treated as hidden variables and the optimality criterion is to maximize the joint likelihood of labeled and unlabeled data. There has been significant previous work on semi-supervised learning, with notable successes; however, there also exist practical examples of performance degradation with the EM approach for semi-supervised learning. For example, Shahshahani et al. [4] describe degradation in image understanding by using Gaussian mixture models (GMM), while Nigam et al. [5] report degradation in naive Bayes classifiers for text classification, and Inoue et al. [7] observe degradation in HMM classifiers. Performance degradation has been principally attributed to model deviations and numerical instability [4]-[6]. Focusing on model deviations, Cozman et al. [6] analyze the degradation by examining asymptotic behavior of the maximum likelihood estimator. They state that when the model is correct the unlabeled data reduce classification error, while when the model is incorrect the role of unlabeled data becomes complex and often results in degradation. On the other hand, Nigam et al. [5] speculate numerical problems in the EM algorithm, and suggest reducing the degradation by weighting the contribution from the unlabeled data, using algorithms such as the so-called EM- $\lambda$ algorithm. However, the choice of suitable scalar parameter $\lambda$ remains an important issue.

Our approach principally follows the previous work of Corduneanu and Jaakkola [14], who used the homotopy method [15]-[17] for semi-supervised learning, for choosing the parameter $\lambda \in$ $[0,1)$ that yields a proper balance between the use of labeled and unlabeled data. In this method semi-supervised learning is regarded as a problem of balancing heterogeneous data sources, with the allocation $\lambda \in[0,1)$ representing the relative balance of labeled data and unlabeled data, where $\lambda=0$ corresponds to purely supervised learning, and $\lambda=1$ to purely unsupervised. The proper balance $\lambda$ is sought as the algorithm gradually morphs the supervised learning problem $(\lambda=0)$ into an unsupervised learning problem $(\lambda=1)$. The associated estimation problem can typically be reduced to solving a set of fixed point equations in the form of a "natural-parameter homotopy" [16].

Being the first to extend this technique to semi-supervised-learning problems, Corduneanu and Jaakkola [14] applied the homotopy method on relatively simple graphical models, such as naive Bayes and Gaussian mixture models (GMM). Using these models, the supervised learning problem $(\lambda=0)$ has a unique solution, and (making a reasonable transversality assumption) 
the theory of globally convergent homotopy algorithms [15], [16] provides a strong existence guarantee of a unique smooth nonbifurcating path of fixed points. However, for the case of more general graphical models (e.g., HMMs), the supervised solution is no longer unique and is rather characterized by multiple local optimums. We show that the theory of globally convergent probability-one homotopy algorithms can be tailored to this more general setting, and a smooth nonbifurcating path of fixed points from $\lambda=0$ to $\lambda=1$ can be identified. We also demonstrate that this framework allows a means of using the unlabeled data to select from among the multiple $\operatorname{HMM} \lambda=0$ (supervised) solutions.

The remainder of the paper is organized as follows. Section II presents a brief introduction to the homotopy method, along with three globally convergent probability-one homotopy maps for the problem of interest here. Section III formulates the optimality criterion of semi-supervised learning for generative models from a mutual information perspective, from which the source balancing problem is introduced. With this background, Sec. IV details the homotopy method for semi-supervised HMM training, with a maximum entropy criterion for choosing the proper $\lambda$. We also discuss how to use unlabeled data to select from among multiple $\lambda=0 \mathrm{HMM}$ solutions. Experimental results on measured and synthetic data are provided in Sec. V, followed in Sec. VI by conclusions and a discussion of future work.

\section{Globally Convergent Probability-One Homotopy Method}

The theory of globally convergent probability-one homotopy maps concerns finding zeros or fixed points of nonlinear systems of equations [15], [16]. The underlying idea is simple: given a twice continuously differentiable function $F: \mathbb{R}^{n} \rightarrow \mathbb{R}^{n}$ of which a zero is sought, rather than solving the original difficult problem $F(z)=0$ directly, start from an "easy" problem $G(z)=0$ whose solution is readily identified, and gradually transform the "easy" problem into the original one, tracking the solutions along the transformation. Typically, one may choose a convex homotopy map such as

$$
H(\lambda, z)=(1-\lambda) G(z)+\lambda F(z)
$$


and trace an implicitly defined zero $\operatorname{curve}^{1} \gamma \in H^{-1}(0)$ from a starting point $(0, z)$ to a final point $(1, \bar{z})$. If this succeeds, then a zero point $\bar{z}$ of $F$ is obtained.

Generally, there are two issues with respect to the homotopy method: (i) whether we can be assured that there exists a smooth path of solutions starting from $\lambda=0$ and reaching a target solution at $\lambda=1$ in finite arc length, and (ii) development of numerical techniques for tracing this path. In the following, we discuss three special homotopy maps that assure the properties desired in (i), with probability one; issue (ii) is also discussed below.

We first consider the globally convergent probability-one fixed point homotopy map

$$
H(\lambda, z)=(1-\lambda)(z-a)+\lambda(z-f(z))
$$

where $a \in \mathbb{R}^{n}$ is a constant vector. The following theorem [16] guarantees under a mild condition the existence of a path reaching a fixed point $\bar{z}$ of $f$.

Theorem 1: Suppose that $B \subset \mathbb{R}^{n}$ is a compact, convex subset, and $f: B \rightarrow B$ is twice continuously differentiable. Then for almost all vectors $a \in$ int $B$, there is a zero path $\gamma$ of $H$ emanating from $(0, a)$, along which the $n \times(n+1)$ Jacobian matrix $D H(\lambda, z)$ has full rank, that does not intersect itself and is disjoint from any other zeros of $H$. $\gamma$ has an accumulation point $(1, \bar{z})$ for which $f(\bar{z})=\bar{z}$. Furthermore, if the Jacobian matrix $D H(1, \bar{z})$ is nonsingular, then the zero path $\gamma$ between $(0, a)$ and $(1, \bar{z})$ has finite arc length.

The second homotopy map deals with a more general case in which $a$ is a function of $z$ and a parameter vector $b$, i.e., $a(b, z)$. The corresponding homotopy map becomes

$$
H(b, \lambda, z)=(1-\lambda)(z-a(b, z))+\lambda(z-f(z))
$$

where the parameter vector $b$ is crucial for the probability-one homotopy theory, as shown in Theorem 2, with proof presented in Appendix I.

Theorem 2: Let $B \subset \mathbb{R}^{n}$ be a compact, convex set with nonempty interior, $U \subset \mathbb{R}^{m}$ a nonempty open set, let $f: B \rightarrow B$ and $a: U \times B \rightarrow$ int $B$ be $C^{2}$ maps, and assume that rank $D_{b} a(b, z)=n$

\footnotetext{
${ }^{1}$ The symbol $H^{-1}(0)$ denotes the set of points $(\lambda, z)$ that satisfy $H(\lambda, z)=0$.
} 


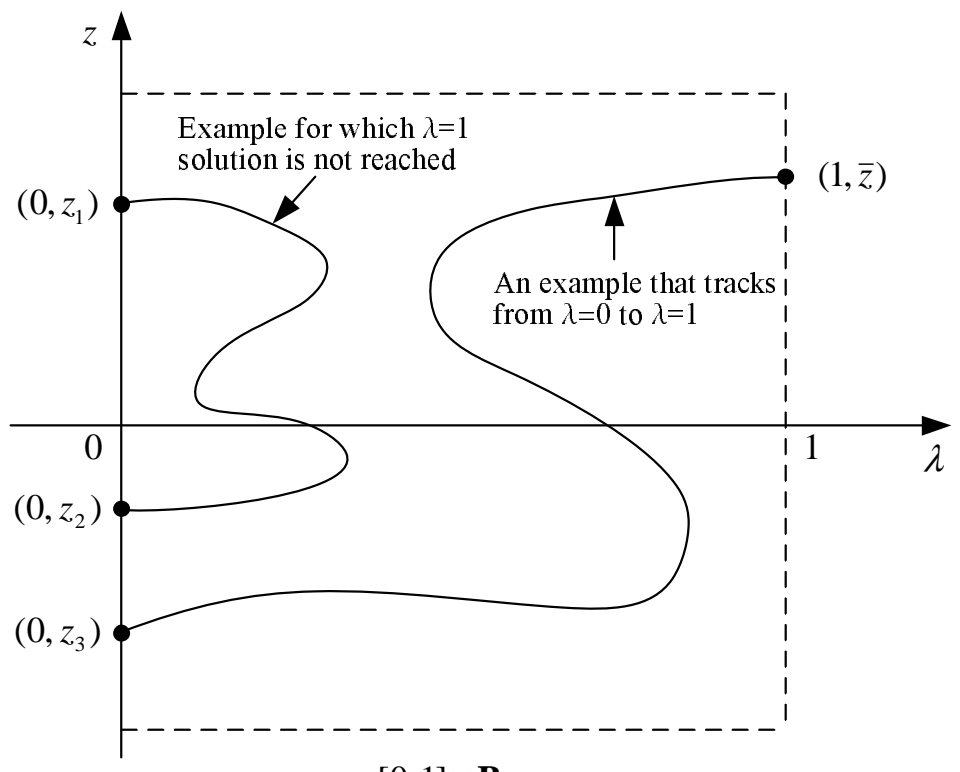

$[0,1] \times \mathbf{B}$

Fig. 1. Zero curve $\gamma$ may return back to $\lambda=0$ and there may not exist a path starting from some fixed points at $\lambda=0$ and reaching a target solution at $\lambda=1$.

for all $(b, z) \in U \times B$. Further assume that for each $b \in U, a_{b}(z)=a(b, z)$ has a unique fixed point $z_{b}$ at which rank $\left(I-D a_{b}\left(z_{b}\right)\right)=n$. Define $H: U \times[0,1) \times B \rightarrow \mathbb{R}^{n}$ by

$$
H(b, \lambda, z)=(1-\lambda)(z-a(b, z))+\lambda(z-f(z))
$$

and define $H_{b}(\lambda, z)=H(b, \lambda, z)$. Then for almost all $b \in U$ there is a zero curve $\gamma$ of $H_{b}(\lambda, z)$ emanating from $\left(0, z_{b}\right)$, along which the Jacobian matrix $D H_{b}(\lambda, z)$ has full rank, that does not intersect itself or other zeros of $H_{b}(\lambda, z)$, and reaches (accumulates at) a fixed point $\bar{z}$ of $f$ at $\lambda=1$. Furthermore, if rank $(I-D f(\bar{z}))=n$, then $\gamma$ has finite arc length.

The above theorem assumes that for each $b \in U, a_{b}(z)=a(b, z)$ has a unique fixed point. If this condition does not hold, i.e., $a_{b}(z)$ has multiple fixed points, then as shown in the proof, it is not guaranteed that a fixed point $\bar{z}$ of $f$ at $\lambda=1$ can be reached; the zero curve $\gamma$ may start from one fixed point of $a_{b}(z)$ and return back to another fixed point of $a_{b}(z)$ at $\lambda=0$, as illustrated in Fig. 1; this is particularly relevant for the HMM, which generally is characterized by multiple $\lambda=0$ (supervised) solutions (as discussed in Sec. IV). 
We therefore consider the homotopy map

$$
H(\lambda, z)=(1-\tanh 60 \lambda)\left(z-a_{0}\right)+\tanh 60 \lambda[(1-\lambda)(z-a(z))+\lambda(z-f(z))]
$$

where $a_{0}$ is a constant vector, and $\tanh (\cdot)$ is the hyperbolic tangent function; the specific numbers in (4) may be altered slightly and still yield similar performance. The point of (4) is that at $\lambda=0$, $H(\lambda, z)$ behaves as $z-a_{0}$ where $a_{0}$ is a constant, as in (2), while as $\lambda \rightarrow 1, H(\lambda, z)$ behaves as (3). The $a_{0}$ in (4) is one of potentially multiple fixed points of $a(z)$. As discussed further below, the homotopy map (4) plays a key role in semi-supervised HMM training, as well as in finding a preferential local optimum for the supervised learning. Technically $\tanh 60 \lambda \neq 1$ at $\lambda=1$, but $\lim _{\lambda \rightarrow 1^{-}} \tanh (60 \lambda /(1-\lambda))=1$ exactly, and for $\lambda \approx 0, \tanh 60 \lambda \approx \tanh (60 \lambda /(1-\lambda))$.

Concerning the second issue addressed above, i.e., numerically tracking the zero path $\gamma$, there are three principal approaches for algorithmic implementations [16]: solving an implicit ordinary differential equation, solving a rectangular system of equations (normal flow), or solving a square system of equations (augmented Jacobian matrix). In all the algorithms, the zero curve $\gamma=$ $(\lambda(s), z(s))$ of $H$ is parameterized by arc length $s$, and the algorithms essentially compute points along $\gamma$ by a predictor-corrector approach to solving $H(\lambda(s), z(s))=0$. All three algorithms can be found in HOMPACK90 [16], a suite of Fortran 90 codes for globally convergent homotopy algorithms.

\section{Semi-Supervised Generative Models And Mutual Information}

Assume data (labeled and unlabeled) are generated from an underlying joint density $p(\boldsymbol{x}, y \mid \Theta)$, where $\boldsymbol{x}$ is the feature vector, $y \in\{1,2, \cdots, C, u\}$ is the corresponding class label and $\Theta$ denotes the model parameters. For sequential data, $\boldsymbol{x}$ represents the concatenation of a sequence of feature vectors. Note that a new category $u$ augments the $C$ classes, to denote a special class of data whose label is missing. Further, assume that the data are generated by the following two steps: (i) select a class according to the class prior probability $p(y \mid \Theta)$, and then (ii) generate a classdependent feature vector $\boldsymbol{x}$ with distribution $p(\boldsymbol{x} \mid y, \Theta)$. For the case $y=u$, which means the label is missing (the corresponding feature vector is unlabeled), the feature vector $\boldsymbol{x}$ is generated

from the marginal density $p(\boldsymbol{x} \mid y=u, \Theta)=\sum_{y=1}^{C} p(\boldsymbol{x}, y \mid y \neq u, \Theta)$. Denote the probability of a 
data missing its label as $p(y=u \mid \Theta)=\lambda$. Then, the likelihood of the model parameters $\Theta$ to have generated $(\boldsymbol{x}, y)$ can be expressed as

$$
p(\boldsymbol{x}, y \mid \Theta)=[(1-\lambda) p(\boldsymbol{x}, y \mid y \neq u, \Theta)]^{\delta(y \neq u)} \times[\lambda p(\boldsymbol{x} \mid y=u, \Theta)]^{\delta(y=u)},
$$

where $\delta(e)=1$ if $e$ is true, and $\delta(e)=0$ otherwise.

The goal is to estimate $\Theta$ from multiple samples $(\boldsymbol{x}, y)$, some of which may be unlabeled. This may be expressed in an information-theoretic setting in terms of the mutual information [18]:

$$
M I(\boldsymbol{x}, y ; \Theta)=\operatorname{En}(\boldsymbol{x}, y)-E n(\boldsymbol{x}, y \mid \Theta),
$$

where $E n$ is the Shannon entropy. $\Theta^{*}$ is sought to maximize the mutual information between samples of $(\boldsymbol{x}, y)$ and the model parameters $\Theta$, i.e., to minimize $\operatorname{En}(\boldsymbol{x}, y \mid \Theta)$ or maximize $E[\log p(\boldsymbol{x}, y \mid \Theta)]$, where in principle the expectation is over $\boldsymbol{x}, y$, and $\Theta$. In a maximum-likelihood (ML) setting for estimation of $\Theta$, the probability density function of $\Theta$ is approximated by a point estimate, i.e., $p(\Theta)=\delta\left(\Theta=\Theta^{*}\right)$, with $\Theta^{*}$ representing the ML parameters. Then using (5) gives

$$
\begin{aligned}
E[\log p(\boldsymbol{x}, y \mid \Theta)]= & E\{\delta(y \neq u)[\log (1-\lambda)+\log p(\boldsymbol{x}, y \mid y \neq u, \Theta)] \\
& +\delta(y=u)[\log \lambda+\log p(\boldsymbol{x} \mid y=u, \Theta)]\} \\
= & (1-\lambda) E[\log p(\boldsymbol{x}, y \mid y \neq u, \Theta)]+\lambda E[\log p(\boldsymbol{x} \mid y=u, \Theta)] \\
& +\lambda \log \lambda+(1-\lambda) \log (1-\lambda)
\end{aligned}
$$

and $\Theta^{*}$ is sought to maximize

$$
J_{\lambda}(\Theta)=(1-\lambda) E[\log p(\boldsymbol{x}, y \mid y \neq u, \Theta)]+\lambda E[\log p(\boldsymbol{x} \mid y=u, \Theta)] .
$$

With labeled data $\left\{\left(\boldsymbol{x}_{1}, y_{1}\right), \cdots,\left(\boldsymbol{x}_{L}, y_{L}\right)\right\}$, and unlabeled data $\left\{\boldsymbol{x}_{L+1}, \cdots, \boldsymbol{x}_{L+U}\right\},(8)$ may be approximated by

$$
J_{\lambda}(\Theta)=(1-\lambda) \frac{1}{L} \sum_{i=1}^{L} \log p\left(\boldsymbol{x}_{i}, y_{i} \mid \Theta\right)+\lambda \frac{1}{U} \sum_{i=L+1}^{L+U} \log p\left(\boldsymbol{x}_{i} \mid \Theta\right),
$$

where the expectations are replaced by the empirical estimate. Moreover, with $\lambda^{*}=U /(L+U)$, the empirical estimation of $p(y=u \mid \Theta)$, the objective function (9) reduces to the traditional optimality criterion for semi-supervised learning [3], [5], [7]:

$$
J_{\lambda^{*}}(\Theta)=\sum_{i=1}^{L} \log p\left(\boldsymbol{x}_{i}, y_{i} \mid \Theta\right)+\sum_{i=L+1}^{L+U} \log p\left(\boldsymbol{x}_{i} \mid \Theta\right)
$$


which jointly maximizes the log-likelihood of labeled data and unlabeled data.

The problem with this choice of $\lambda$ is that for cases of limited labeled data (typically, $U \gg L$ ) the algorithm almost becomes unsupervised, and therefore the labeled data (arguably the most useful data) are almost unused in a relative sense. A solution should be sought with another allocation $\lambda \in[0,1)$ between a purely supervised learning $(\lambda=0)$ and a purely unsupervised learning $(\lambda=1)$. In the following, we focus on solving (9) considering all $\lambda$ from $\lambda=0$ to $\lambda=1$. As discussed further below, a maximum entropy criterion is then used to evaluate the solutions along this full path for choosing a proper $\lambda$.

A general approach to optimize (9), for a given $\lambda$, is to use the EM algorithm [13]. In this procedure, the labels of the unlabeled data are treated as hidden variables, and the algorithm iteratively updates the model parameters $\Theta$ via the $\mathrm{E}$ step and the $\mathrm{M}$ step, until convergence to a local optimum. Typically, one may regard each EM iteration as a fixed point iteration of the form $\Theta^{t+1}=E M_{\lambda}\left(\Theta^{t}\right)$, which acts on the parameters of the current estimate $\Theta^{t}$ and produces another estimate $\Theta^{t+1}$ that monotonically increases the likelihood. A converged solution of the EM algorithm is a fixed point of an EM operator. Since the homotopy method can be used to find the fixed points of a nonlinear system of equations, this point of view motivates applying the homotopy method to the EM operator.

The detailed fixed point EM operator for solving (9) depends on the model used. In the following, we consider the case of HMMs for semi-supervised training, and use the homotopy method to track the fixed point solutions from $\lambda=0$ to $\lambda=1$.

\section{Semi-Supervised LEARNing OF HMMs Via A Homotopy Method}

\section{A. HMM classifier}

Using similar notation as in [8], we define an $N$-state discrete HMM with an observation alphabet size of $M$, parameterized as $\theta=\left\{\pi^{N}, A^{N \times N}, B^{N \times M}\right\}$, where $\pi$ is the initial-state probability vector, $A$ is the state-transition matrix, and $B$ is the observation matrix (probability of observing each of the $M$ alphabet members in a particular state). Then given an observation sequence 
$\boldsymbol{x}=\left\{x_{1}, \cdots, x_{T}\right\}$, the likelihood of the model $\theta$ is calculated as

$$
p(\boldsymbol{x} \mid \theta)=\sum_{s} \pi_{s_{1}} \cdot \prod_{t=1}^{T-1} a_{s_{t} s_{t+1}} \cdot \prod_{t=1}^{T} b_{s_{t}}\left(x_{t}\right),
$$

where $s=\left(s_{1}, \cdots, s_{T}\right)$ is the hidden state sequence, and the summation is over all possible state sequences.

For a sequential data classification task, an HMM classifier is built to assign a class label for a given data sequence. Based on the generative assumption in Sec. III, in this case each class will, in general, be modeled as an HMM with a distinct set of parameters, i.e., $\theta^{y}=\left\{\pi^{y}, A^{y}, B^{y}\right\}$, for each $y \in\{1,2, \cdots, C\}$. By incorporating the class prior distribution $\left\{w^{1}, \cdots, w^{C}\right\}$, the posterior of class label $y$ can be computed via Bayes rule as

$$
p(y \mid \boldsymbol{x}, \Theta)=\frac{w^{y} p\left(\boldsymbol{x} \mid \theta^{y}\right)}{\sum_{y^{\prime}=1}^{C} w^{y^{\prime}} p\left(\boldsymbol{x} \mid \theta^{y^{\prime}}\right)}
$$

where $0 \leq w^{y} \leq 1$ for all $y \in\{1,2, \cdots, C\}$, and $\sum_{y=1}^{C} w^{y}=1$, and $\Theta=\left\{w^{1}, \theta^{1}, \cdots, w^{C}, \theta^{C}\right\}$ denotes the cumulative parameters of the HMM classifier. The class that has the highest posterior probability is used as the estimated class label.

\section{B. Semi-supervised HMM training via a homotopy method}

Given the labeled data sequences $\left\{\left(\boldsymbol{x}_{1}, y_{1}\right), \cdots,\left(\boldsymbol{x}_{L}, y_{L}\right)\right\}$ and unlabeled data sequences $\left\{\boldsymbol{x}_{L+1}\right.$, $\left.\cdots, x_{L+U}\right\}$, the parameters of the HMM classifier $\Theta$ can be estimated by maximizing the objective function (9), which can be implemented by the homotopy method on the fixed point EM operator, as discussed in Sec. III.

Applying the EM algorithm to semi-supervised learning of HMMs, i.e., to objective function (9), is straightforward, and the resulting algorithm is an extension of the standard Baum-Welch algorithm to handle the unlabeled data (e.g., see [7]). This yields the fixed point equations as follows:

$$
\begin{aligned}
& \tilde{w}^{y}=(1-\lambda) \frac{1}{L} \sum_{i=1}^{L} \delta\left(y=y_{i}\right)+\lambda \frac{1}{U} \sum_{j=L+1}^{L+U} p\left(y \mid \boldsymbol{x}_{j}, \Theta\right) \\
& \tilde{\pi}_{k}^{y}=(1-\lambda) \frac{1}{L} \sum_{i=1}^{L} \gamma_{1}^{i}(k) \delta\left(y=y_{i}\right)+\lambda \frac{1}{U} \sum_{j=L+1}^{L+U} \gamma_{1}^{j}(k) p\left(y \mid \boldsymbol{x}_{j}, \Theta\right),
\end{aligned}
$$




$$
\begin{aligned}
\tilde{a}_{k l}^{y}=(1-\lambda) \frac{1}{L} \sum_{i=1}^{L}\left[\sum_{t=1}^{T-1} \xi_{t}^{i}(k, l)\right] \delta\left(y=y_{i}\right) & \\
& +\lambda \frac{1}{U} \sum_{j=L+1}^{L+U}\left[\sum_{t=1}^{T-1} \xi_{t}^{j}(k, l)\right] p\left(y \mid \boldsymbol{x}_{j}, \Theta\right), \\
\tilde{b}_{k}^{y}(v)=(1-\lambda) \frac{1}{L} \sum_{i=1}^{L} & {\left[\sum_{t=1}^{T} \gamma_{t}^{i}(k) \delta\left(x_{i, t}=v\right)\right] \delta\left(y=y_{i}\right) } \\
& +\lambda \frac{1}{U} \sum_{j=L+1}^{L+U}\left[\sum_{t=1}^{T} \gamma_{t}^{j}(k) \delta\left(x_{j, t}=v\right)\right] p\left(y \mid \boldsymbol{x}_{j}, \Theta\right),
\end{aligned}
$$

where the left hand sides of the above equations are unnormalized parameters of an HMM classifier, related to $\Theta$ by

$$
\pi_{k}^{y}=\frac{\tilde{\pi}_{k}^{y}}{\sum_{i} \tilde{\pi}_{i}^{y}}
$$

with $\tilde{\pi}_{i}^{y} \geq 0$, for all $i \in\{1, \cdots, N\}, y \in\{1, \cdots, C\}$, and similarly for the parameters $w^{y}, a_{k l}^{y}$ and $b_{k}^{y}(v)$. With $\lambda=0$, fixed point equations (13)-(16) after normalization degenerate to the standard Baum-Welch algorithm [8] for supervised HMM parameter updating.

Summarizing the HMM fixed point equations (13)-(16), we may write them in matrix form as:

$$
H(\lambda, \tilde{\Theta})=(1-\lambda)\left(\tilde{\Theta}-E M_{0}(\Theta)\right)+\lambda\left(\tilde{\Theta}-E M_{1}(\Theta)\right)=0
$$

where $\tilde{\Theta}=\left\{\tilde{w}^{1}, \tilde{\theta}^{1}, \cdots, \tilde{w}^{C}, \tilde{\theta}^{C}\right\}$ is an unnormalized version of $\Theta, E M_{0}(\Theta)$ are the right hand side terms of (13)-(16) when $\lambda=0$, and $E M_{1}(\Theta)$ are the right hand side terms of (13)-(16) when $\lambda=1$. Note that (18) is in the same form as (3), forming a "natural-parameter homotopy" (where here $\tilde{\Theta}$ represents the variable $z$ used when introducing the homotopy method, and $b$ corresponds to choosing a fixed point of $E M_{0}(\Theta)$ ).

We emphasize that the EM algorithm is employed for supervised HMM training $(\lambda=0)$ with the state sequences treated as the hidden variables. For semi-supervised HMM training the EM algorithm becomes more involved, since in this case there are two levels of hidden variables: one is the hidden state sequences for the labeled and unlabeled data, and the other is the hidden labels of the unlabeled data. Because of the optimization with hidden variables, both the supervised solution $(\lambda=0)$ and the unsupervised solution $(\lambda=1)$ are characterized by multiple local optimums. Based on the discussion in Sec. II, in this case if we use the homotopy map (3) for path tracking, it is not guaranteed that a fixed point solution at $\lambda=1$ can be reached, and the 
zero curve $\gamma$ may start from one fixed point of $E M_{0}(\Theta)$ and return back to another fixed point of $E M_{0}(\Theta)$ at $\lambda=0$ (see Fig. 1). For this reason, when we implement the homotopy method for semi-supervised HMM training, we use the homotopy map (4), which is a close approximation to (3) but with a strong existence theorem assuring tracking from $\lambda=0$ to $\lambda=1$.

Transforming (18) into the form of (4), we obtain

$$
\begin{aligned}
H(\lambda, \tilde{\Theta})= & (1-\tanh 60 \lambda)\left(\tilde{\Theta}-\Theta_{0}\right) \\
& +\tanh 60 \lambda\left[(1-\lambda)\left(\tilde{\Theta}-E M_{0}(\Theta)\right)+\lambda\left(\tilde{\Theta}-E M_{1}(\Theta)\right)\right],
\end{aligned}
$$

where $\Theta_{0}$ is a constant vector, which we here set to a fixed point of $E M_{0}(\Theta)$, corresponding to selecting a local supervised solution. To implement the homotopy algorithm, the Jacobian matrices $\nabla_{\tilde{\Theta}} E M_{0}(\Theta)$ and $\nabla_{\tilde{\Theta}} E M_{1}(\Theta)$ are required, which subsequently require the partial derivatives of $\gamma_{t}(i), \xi_{t}(i, j)$ and $p(y \mid \boldsymbol{x}, \Theta)$ with respect to each element of $\tilde{\Theta}$. However, these partial derivatives are not computationally feasible for the currently available variables in HMMs, such as $\gamma_{t}(i)$ and $\xi_{t}(i, j)$ [8]. We thus define a set of new variables $\psi, \phi$, and $\Phi$, as presented in Appendix II, to facilitate computation of the Jacobian matrices. Finally, we summarize the computational formulas for $\nabla_{\tilde{\Theta}} E M_{0}(\Theta)$ and $\nabla_{\tilde{\Theta}} E M_{1}(\Theta)$ in Appendix III.

We note that for the relatively simple models, such as naive Bayes and Gaussian mixture models (GMM), as used by Corduneanu and Jaakkola in [14], the supervised solution $(\lambda=0)$ is unique. Therefore, the associated fixed point equations can be formulated as a "natural-parameter homotopy" as in (2), whose success is guaranteed by Theorem 1. From this point of view, our application of the homotopy map (4) generalizes the previous work of Corduneanu and Jaakkola to a more general case of semi-supervised learning for generative models.

\section{Determination of parameter $\lambda$}

As discussed in Sec. II, the homotopy map (4) is an approximation to (3), overcoming the multiple local optimal solutions of the supervised HMM, and assuring with probability one a track from $\lambda=0$ to $\lambda=1$. While we have found this successful track to be the case for the HMM, interesting numerical phenomena have been observed, as demonstrated in Sec. V. Specifically, for many $\lambda=0$ initializations, each representing a particular supervised (local optimal) HMM 
solution, we observe a homotopy zero curve tracking away from $\lambda=0$ and then backtracking to the vicinity of another $\lambda=0$ solution, which we attribute to a different local optimal supervised solution (see Fig. 2(a)). The $\lambda$ then increases away from this local optimal supervised solution (at $\lambda=0$ ), often followed by tracking back to near a different $\lambda=0$ solution. This "hopping" between different supervised solution neighborhoods (at $\lambda=0$ ) often occurs many times, before there is a final departure of the homotopy zero curve track from the last visited $\lambda=0$ solution neighborhood, followed by a smooth track to a $\lambda=1$ (unsupervised) solution.

We interpret this phenomenon as follows. The supervised HMM analysis permits multiple (local optimal) solutions. For cases of limited labeled data, it is anticipated that there may be more such local optimal solutions, with enhanced uncertainty as to which is appropriate. When $\lambda$ moves away from $\lambda=0$ in the homotopy zero curve track, the algorithm begins to sense the properties of the (abundant) unlabeled data. If the $\lambda=0$ solution corresponds to a supervised solution that is inconsistent with the unlabeled data, the homotopy zero curve tracks back to near a different $\lambda=0$ solution that is better matched to the unlabeled data. This tracking away from $\lambda=0$ solution neighborhoods and back to different $\lambda=0$ solution neighborhoods may occur multiple times before an appropriate $\lambda=0$ solution neighborhood is found, after which the homotopy method tracks smoothly to the unsupervised $\lambda=1$ solution.

This interpretation, which we support with data in Sec. V, indicates that the homotopy method is selecting a supervised $(\lambda=0)$ solution (by visiting its neighborhood) that is well matched to the unlabeled data. As discussed in Sec. V, the parameters associated with this $\lambda=0$ solution may therefore be desirable parameters for the HMM classifiers of interest. In addition, one may wish to choose a $\lambda \in[0,1)$ at which to operate for learning the HMM parameters, rather than simply selecting from the multiple $\lambda=0$ solutions. In this case we select $\lambda$ based on an analysis of the final complete track to $\lambda=1$, from the selected $\lambda=0$ solution, since based on the above discussion this track constitutes the case of an appropriate match between the supervised and unsupervised solutions.

As discussed above, using the homotopy map (4) for the HMM, the algorithm often appears to "hop" between different $\lambda=0$ supervised solution neighborhoods before executing a smooth (monotone increasing in $\lambda$ ) track to the unsupervised $\lambda=1$ solution. This final complete track 
(zero curve segment) appears to be one for which the local supervised $(\lambda=0)$ and unsupervised $(\lambda=1)$ solutions are in most agreement. We therefore consider determination of an "appropriate" $\lambda$ value by considering solution properties along this final path from the vicinity of $\lambda=0$ to $\lambda=1$; refer to this final path segment to $\lambda=1$ as $P_{F}$.

For each $\lambda$ along $P_{F}$, we have a fixed point solution for the parameters of the HMM classifier $\Theta_{\lambda}$. Therefore, for each $\lambda$ we may compute the average uncertainty in the labels of the unlabeled data, quantified via the entropy

$$
\operatorname{En}\left(Y \mid \Theta_{\lambda}\right)=-\frac{1}{U} \sum_{i=L+1}^{L+U} \sum_{y=1}^{C} p\left(y \mid \boldsymbol{x}_{i}, \Theta_{\lambda}\right) \log p\left(y \mid \boldsymbol{x}_{i}, \Theta_{\lambda}\right) .
$$

As demonstrated in Sec. V, we have found that the $\lambda$ that maximizes (20) along $P_{F}$ provides a good estimate for the parameters of the HMM classifier. The $\lambda$ that maximizes (20) implies a point of greatest classification uncertainty, which we attribute to a point at which the balance between labeled and unlabeled data introduces the least classification confidence. This is analogous to the analysis performed in [14], in which the $\lambda$ operating point was determined as the point at which there was an apparent transition between the purely supervised and purely unsupervised solutions. As demonstrated in Sec. V this selection procedure for $\lambda$ has yielded good performance on the data considered thus far, but further investigation of this measure is warranted.

\section{EXPERIMENTAL RESULTS}

We test the performance of the homotopy method on measured acoustic data and synthetic data. We use the homotopy method to select a local supervised solution that appears to be consistent with the unlabeled data, and we also seek a $\lambda \in[0,1)$ for semi-supervised learning, with the latter found on the zero curve track segment $P_{F}$ discussed above.

Based on previous studies, we anticipate that the conventional semi-supervised solution will work well for synthetic data, for which the model fits perfectly [6]. However, for the HMM modeling of measured data, for which the HMM is just a model, the performance of the conventional semisupervised solution is expected to degrade, and it is here that the homotopy results are expected 
to excel. Both the measured data and synthetic data used in the experiments are available at http://www.ee.duke.edu/ I larin/homotopy_HMMs.zip.

\section{A. Experiments on measured data}

We consider a multi-aspect target classification task based on measured acoustic scattering data. Details on using HMMs for this application may be found in [9]. We here provide the basic idea of multi-aspect target classification and a brief description of the characteristics of the measured data. Typically, the acoustic fields scattered from a complex target are a strong function of target-sensor orientation. However, there are often sets of contiguous target-sensor orientations for which the scattering data are relatively stationary, with each such set termed a target "state". When sensing is performed from a sequence of target-sensor orientations, one implicitly senses scattered fields sampled from a sequence of target states. This sequence of sampled states may be modeled as a Markov process [9]. The target states are typically "hidden", since the target is generally distant or concealed, yielding an HMM representation of the scattering data. For each target-sensor orientation the associated acoustic scattered fields are mapped to a feature vector, and then this feature vector is quantized using vector quantization (VQ) [19]. The sequence of measured scattered waveforms is therefore mapped to a sequence of code indices, modeled via a discrete HMM. Details on the targets and on the feature extraction employed may be again found in [9].

Each target is modeled as a 2-state HMM with an observation alphabet size of 10. The task concerns classifying between two targets based on a sequence of 8 observations (corresponding in the physical problem to viewing the target from eight orientations, at $5^{\circ}$ angular sampling, for a total aperture of $35^{\circ}$ ). The original data are sampled at $1^{\circ}$ angular increment, and therefore there are a total of 360 data sequences for each target, defined by the initial angle of orientation. We randomly select 10 data sequences as the labeled data, and use the remaining 350 data sequences as the unlabeled data. Therefore, there are totally $L=20$ labeled data sequences (10 data sequences for each of the two targets) and $U=700$ unlabeled data sequences, on which a semi-supervised HMM classifier is trained.

We use the normal flow algorithm, as implemented in the subroutine STEPNF from HOM- 


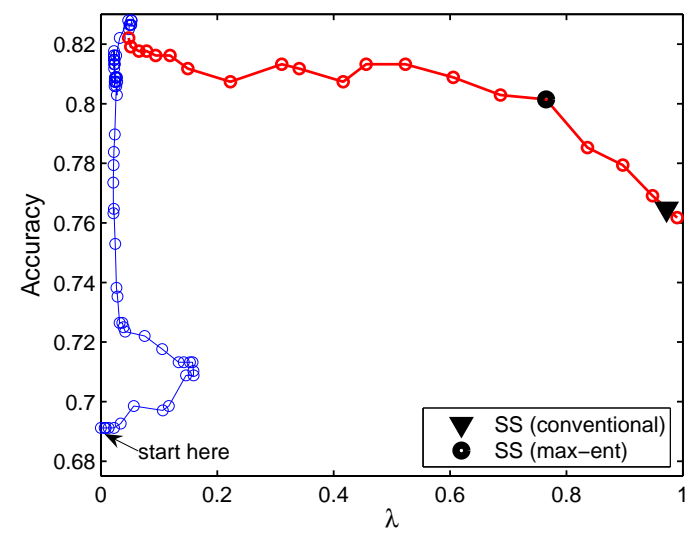

(a)

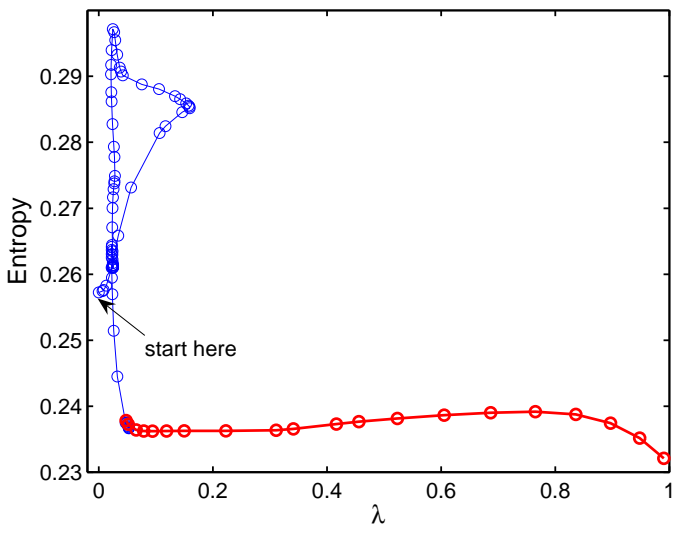

(b)

Fig. 2. Path tracking using the homotopy map (4). The initial part of the path is shown in blue, and the final path segment $P_{F}$ indicated above is shown in red. We indicate in (a) the stop criteria based on a "conventional" $\lambda=U /(L+U)$, as well as based on the condition in (20). (a) Classification accuracy on the unlabeled data as a function of $\lambda$; (b) classification entropy as a function of $\lambda$.

PACK90 [16], to compute the fixed point solutions of (19) for various allocations $\lambda$. Each $\lambda$ along the track is determined by the algorithm that starts at one randomly selected fixed point solution of the supervised HMM and tracks the fixed point solutions along the zero curve $\gamma$. For each obtained fixed point solution (at each reachable $\lambda$ ), we obtain the parameters of the HMM classifier $\Theta_{\lambda}$, via the normalization (17). This classifier is then applied on the unlabeled data to evaluate classification accuracy.

In Fig. 2 we show an example result of the homotopy method for path tracking of the semisupervised HMM. The evolution of classification accuracies as a function of $\lambda$ for the homotopy map (4) are shown in Fig. 2(a), and the entropy from (20) is shown in Fig. 2(b). In Fig. 2 the initial portion of the homotopy path is in blue, and the final path segment $P_{F}$ from the vicinity of $\lambda=0$ to $\lambda=1$ is shown in red. We observe in Fig. 2(a) that the homotopy method initially tracks between different solutions near $\lambda=0$, before completing a path to $\lambda=1$. Note as well that the classification performance for the $\lambda \approx 0$ solutions, as measured on the unlabeled data, increases with successive visits to $\lambda \approx 0$ solutions; it is important to emphasize that in practice this classification performance information would be unavailable and cannot be used to select $\lambda$. 


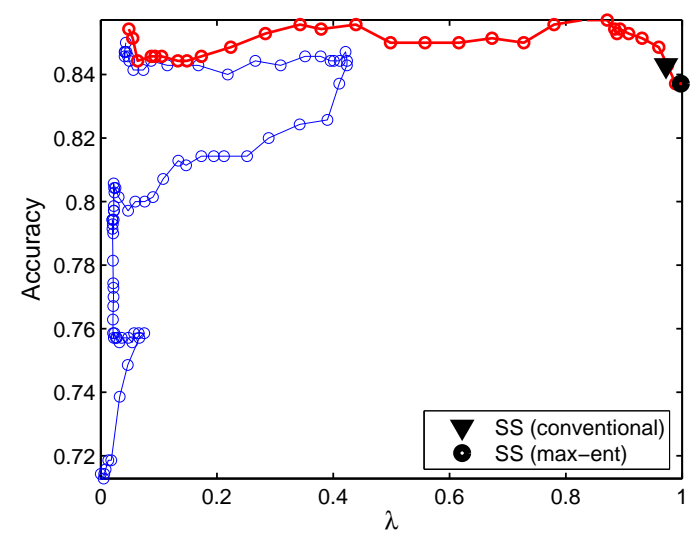

(a)

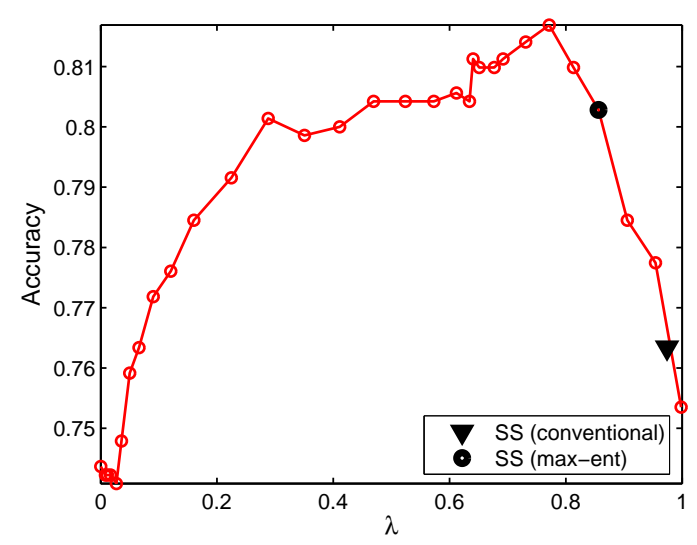

(c)

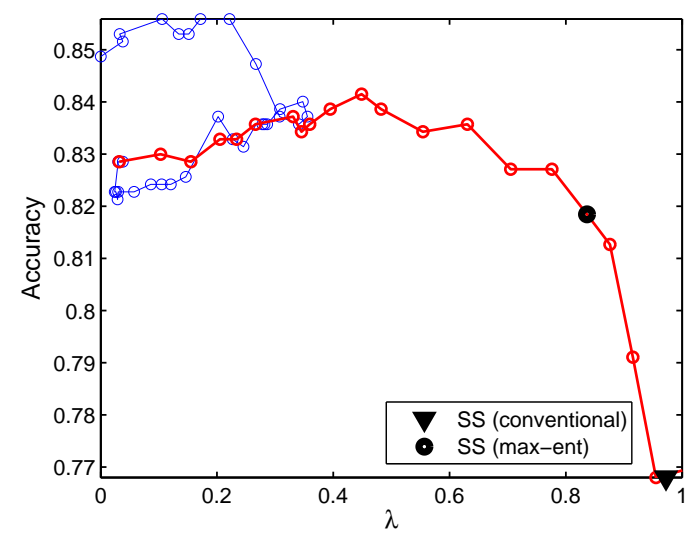

(e)

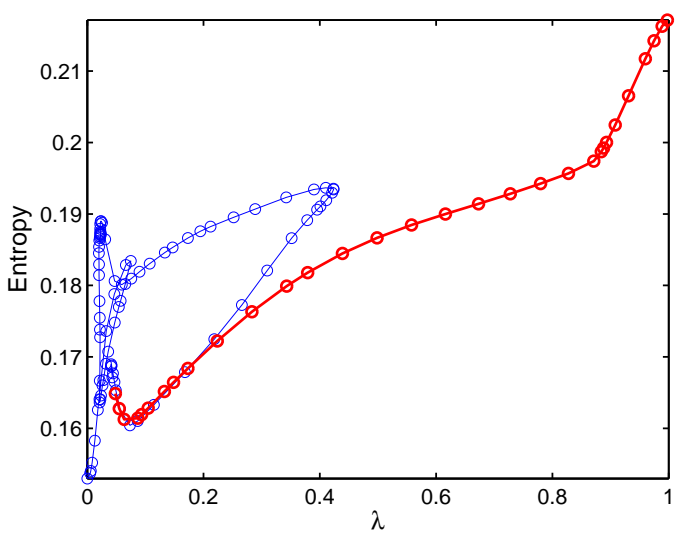

(b)

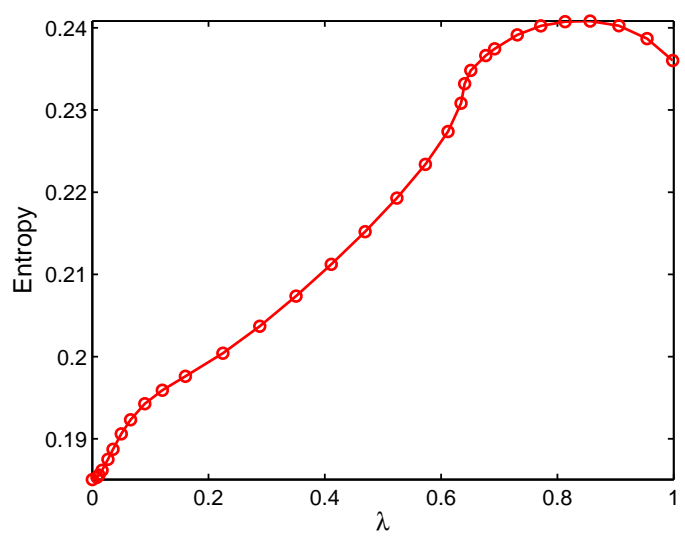

(d)

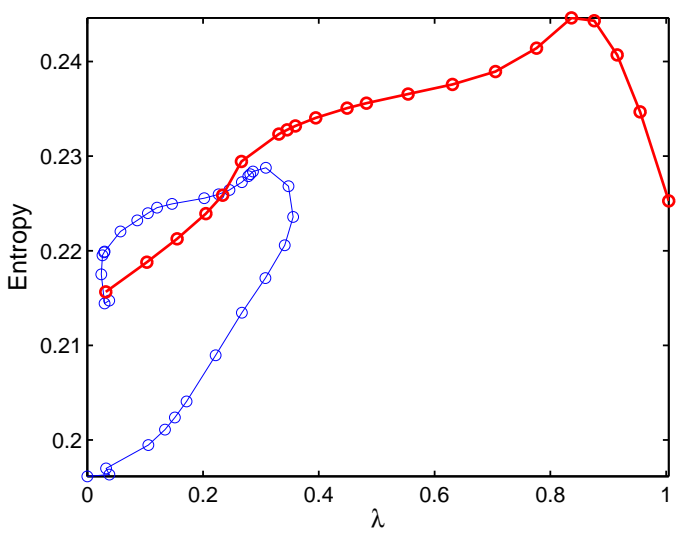

(f)

Fig. 3. Three sets of typical results obtained from 60 runs. The three cases are shown vertically, with the left figure showing the homotopy track vs. accuracy, and the right showing the entropy in (20) as a function of $\lambda$. We indicate in (a), (c) and (e) the stop criteria based on a "conventional", as well as based on the condition in (20). 
The results in Fig. 2(a) simply represent performance that could be achieved for each of the fixed point solutions along the homotopy path. The results in Fig. 2(a) indicate that the last $\lambda \approx 0$ solution, at the start of $P_{F}$, may be a good operating point for the HMMs, corresponding to selection of a particular (local) supervised solution. We do indeed consider supervised HMMs, with EM-determined parameters initialized at values given at the start of $P_{F}$, and this yields good results on average. Although this final HMM solution is purely supervised, the unlabeled data have been used to determine a good EM initialization point, thereby selecting from among the multiple $\lambda=0$ solutions. Using the criterion in (20) we find $\lambda \approx 0.75$, while the "conventional" semi-supervised method chooses $\lambda \approx 0.97$.

Three additional typical examples are shown in Fig. 3, for the same quantity of labeled data, but for different specific data in each class. The top and bottom examples in Fig. 3 are similar to Fig. 2, while the middle example is a case for which the initial $\lambda=0$ solution yields a monotone increasing (in $\lambda$ ) track to $\lambda=1$. For cases like the middle example in Fig. 3, sometimes the $\lambda=0$ solution yields the highest classification accuracy, while other times (like in Fig. 3) this is not the case. We note that in the middle example of Fig. 3 the measure in (20) yields a good estimate for $\lambda$, based on the classification performance.

In Fig. 3(b) we note that the criterion in (20) would select $\lambda \approx 1$, which is essentially an unsupervised solution. This implies that the HMM classifier uses the unsupervised solution to perform classification, with the labels of the two HMM classes known as a result of the homotopy track.

To provide a statistically meaningful analysis, we again consider 10 labeled data per class (20 total), and compute average classification performance and homotopy path characteristics. We consider 60 distinct runs of this case, randomly selecting the labeled and unlabeled examples. In Table I we present average performance relative to the initial supervised solution computed for $\lambda=0$. The results of three different algorithms are presented. In the first we consider a supervised classifier designed using the homotopy method, using HMM parameters computed for the last $\lambda \approx 0$ solution before a direct homotopy track is realized to $\lambda=1$. This solution is supervised, since $\lambda \approx 0$, but the unlabeled data are used via homotopy to select a supervised $(\lambda \approx 0)$ solution. In the second approach we consider a semi-supervised solution using $\lambda=U /(L+U)$, computed 
TABLE I

AVERAGE CLASSIFICATION PERFORMANCE RELATIVE to THE INITIAL $\lambda=0$ SOLUtion. Results ARE AVERAGED OVER A TOTAL OF 60 EXAMPLES, FOR WHICH 10 OF 360 DATA SEQUENCES ARE SELECTED RANDOMLY, AND TREATED AS LABELED DATA, THE REMAINING 350 TREATED AS UNLABELED. FOR THE HOMOTOPY COMPUTATIONS, OF THE 60 CASES 36 EXPERIENCED BACKTRACKING to A $\lambda \approx 0$ SOLUtion, AS IN FIG. 2. AVERAGE RELATIVE ClassifiCATION IMPROVEMENT IS SHOWN FOR THE CASES IN WHICH HOMOTOPY MANIFESTS AT LEAST ONE RETURN TO A $\lambda \approx 0$ SOLUTION, WHEN IT DOES NOT, AND AVERAGE OVERALL PERFORMANCE.

\begin{tabular}{|c|c|c|c|}
\hline & last $\lambda \approx 0$ & $\lambda=U /(L+U)$ & $\lambda_{M E}$ \\
\hline with return to $\lambda \approx 0$ & $4.95 \%$ & $3.10 \%$ & $6.19 \%$ \\
\hline without return to $\lambda \approx 0$ & 0 & $-2.86 \%$ & $0.98 \%$ \\
\hline Total & $3.02 \%$ & $0.72 \%$ & $4.11 \%$ \\
\hline
\end{tabular}

along the final homotopy track $P_{F}$ to $\lambda=1$; this is the "conventional" solution shown in Figs. 2 and 3. Finally, we consider a semi-supervised solution at $\lambda_{M E}$, computed by maximizing (20) along the final homotopy track $P_{F}$ to $\lambda=1$; this is the "max-ent" solution in Figs. 2 and 3.

Of the 60 runs considered (different selections of the 10 labeled data), we observed backtracking of the type in Fig. 2 on 36 cases, where in 24 cases no backtracking of the homotopy solution was observed, as in Fig. 3(c). In Table I we present results when the homotopy does have a return to $\lambda \approx 0$ (backtracking), and when it does not, as well as total average performance. It is observed that, for these data, the $\lambda_{M E}$ semi-supervised solution provides the best improvement relative to the initial supervised solution.

In Fig. 4 we show supervised and semi-supervised results for a more extensive range of labeled samples, using the same data as considered above. The supervised result uses no homotopy, and the two semi-supervised solutions both use homotopy; the latter two are distinguished by how $\lambda$ is determined, where in one case ("conventional") we set $\lambda=U /(L+U)$ and in the other we set $\lambda$ as in (20). The criterion in (20) yields superior performance compared to $\lambda=U /(L+U)$ for these measured data, for which the model is not perfect. 


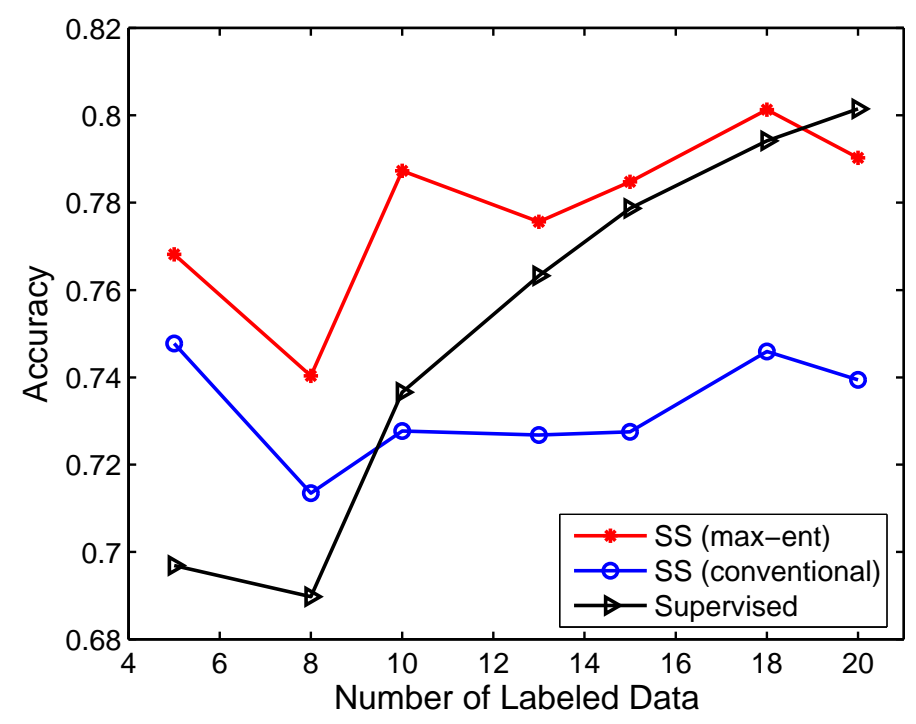

Fig. 4. Classification accuracy on measured data as a function of labeled sequences per target, for the supervised learning (Supervised), the conventional semi-supervised learning (SS (conventional)) with $\lambda=U /(L+U)$, and the homotopy method based on the maximum entropy (SS (max-ent)).

\section{B. Experiments on synthetic data that match the model}

In the last experiment, we test the performance of the homotopy method on a synthetic data set, which includes 2 classes of sequential data where each class has 500 data sequences of length 5. The data are generated with a 2-state HMM with an observation alphabet size of 5. We use the same experimental setting as in Fig. 4, with the results shown in Fig. 5. In this case the data were generated from the same model used for analysis, and good performance is observed when using $\lambda=U /(L+U)$.

The results in Fig. 5 demonstrate that for the case in which the data fits the model, the semisupervised solution with $\lambda=U /(L+U)$ yields the best results on average, while the homotopy based solution based on (20) is slightly worse. Both semi-supervised solutions are significantly better than the supervised solution, particularly for small $L$.

Figs. 4 and 5 show results using the "conventional" means of choosing $\lambda$, at $\lambda=U /(L+U)$. We note that even when using $\lambda=U /(L+U)$ the homotopy method may provide a different 


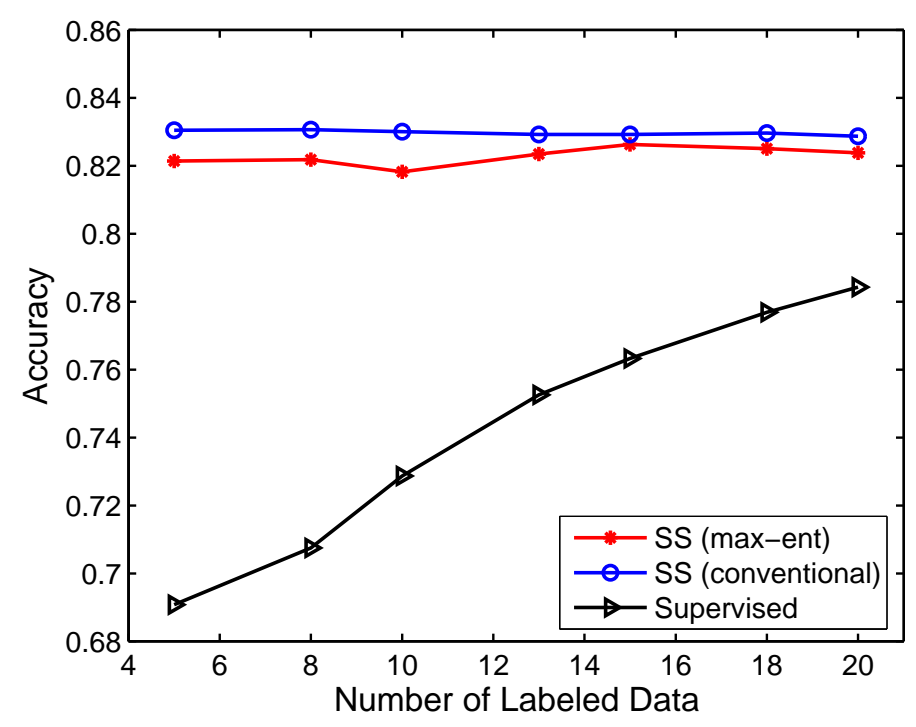

Fig. 5. Classification accuracy on synthetic data as a function of labeled sequences per target, for the supervised learning (Supervised), the conventional semi-supervised learning (SS (conventional)) with $\lambda=U /(L+U)$, and the homotopy method based on the maximum entropy (SS (max-ent)).

solution than previous work in which $\lambda$ was set this way. Specifically, previously research solved (13)-(16) with $\lambda=U /(L+U)$. By contrast, in the homotopy method we track solutions from $\lambda=0$ to $\lambda=1$, and select one particular fixed point solution. Consequently, even when using $\lambda=U /(L+U)$ there is a distinction between the homotopy formulation developed here and previous semi-supervised solutions for HMMs. For the case for which the model matches the data, as in Fig. 5, we have found using $\lambda=U /(L+U)$ in (13)-(16) and within the homotopy formulation yield similar results, while this is not the case when the model does not match perfectly (Fig. 4).

\section{CONCLUSION}

In this paper we have considered the semi-supervised design of HMMs, based on a globally convergent probability-one homotopy method that yields a path of fixed point HMM solutions, each utilizing a different balance of labeled and unlabeled data, dictated by a parameter $\lambda$. A significant challenge involved addressing the multiple local optimal solutions afforded by the 
supervised HMM solution. To address this problem a new probability-one homotopy map was constructed, which allowed analysis of the desired semi-supervised problem, without problems posed by multiple local-optimal supervised solutions.

This homotopy formulation yielded interesting phenomena based on analysis of measured sequential data (for which the model is not perfect). We observed that the homotopy algorithm often tracked away from one supervised $(\lambda=0)$ solution neighborhood to another one, until manifesting a final track from a last supervised solution neighborhood to the purely unsupervised $(\lambda=1)$ solution. We referred to this as the "final" homotopy path segment $P_{F}$. Based on a detailed analysis of performance, we attributed this phenomena to a homotopy-based examination of multiple supervised solutions, until a solution is found that is in agreement with the properties of the unlabeled data, and from this supervised solution neighborhood there is a final track to the unsupervised solution at $\lambda=1$.

Having developed the homotopy method, there is now the issue of choosing from among the numerous fixed point solutions along the homotopy path, with this solution used in the final classification. Three different ways were considered for choosing this single fixed point solution: (i) $\lambda=U /(L+U)$, where $L$ and $U$ represent the number of labeled and unlabeled data, respectively; (ii) using the $\lambda \approx 0$ solution at the beginning of the final homotopy path segment $P_{F}$; and (iii) considering the $\lambda$ along $P_{F}$ that maximizes the classification uncertainty, computed in terms of the entropy. We found that methods (ii) and (iii) yielded superior results compared to (i), when considering data for which there was not a perfect match between the data and model. Methods (ii) and (iii) yielded similar results, although on average (iii) was slightly better.

For models like the HMM, for which there are multiple local supervised solutions, further research is required on selecting the optimal $\lambda$. Specifically, at this point there is limited theoretical justification for method (iii) above. A more detailed theoretical justification for this selection method for $\lambda$ is warranted, as is consideration of other methods for selecting $\lambda$ based on the homotopy path from $\lambda=0$ to $\lambda=1$. 


\section{REFERENCES}

[1] S. J. Raudys and A. K. Jain, "Small sample size effects in statistical pattern recognition: recommendations for practitioners," IEEE Trans. on Pattern Analysis and Machine Intelligence, vol. 13, no. 3, pp. 252-264, Mar. 1991.

[2] X. Zhu, Z. Ghahramani, and J. Lafferty, "Semi-supervised learning using gaussian fields and harmonic functions," in Proc. of The 20th International Conference on Machine Learning, no. 912-919, 2003.

[3] M. Seeger, "Learning with labeled and unlabeled data," Institute for Adaptive and Neural Computation, University of Edinburgh, Edinburgh, UK, Tech. Rep., 2001.

[4] B. Shahshahani and D. Landgrebe, "The effect of unlabeled samples in reducing the small sample size problem and mitigating the Hughes phenomenon," IEEE Trans. on Geosc. and Remote Sensing, vol. 32, 1994.

[5] K. Nigam, A. Mccallum, S. Thrun, and T. Mitchell, "Text classification from labeled and unlabeled documents using EM," Machine learning, vol. 39, pp. 135-167, 2000.

[6] F. G. Cozman, I. Cohen, and M. C. Cirelo, "Semi-supervised learning of mixture models," in Proc. of the 20th International Conference on Machine Learning, 2003.

[7] M. Inoue and N. Ueda, "Exploitation of unlabeled sequences in hidden Markov models," IEEE Trans. on Pattern Analysis and Machine Intelligence, vol. 25, no. 12, 2003.

[8] L. R. Rabiner, "A tutorial on hidden Markov models and selected applications in speech recognition," Proc. IEEE, vol. 77, no. 2, pp. 257-286, 1989.

[9] P. Runkle, P. K. Bharadwaj, L. Couchman, and L. Carin, "Hidden Markov models for multi-aspect target classification," IEEE Trans. Signal Proc., vol. 47, pp. 2035-2040, Jul. 1999.

[10] E. Birney, "Hidden Markov models in biological sequence analysis," IBM Journal of Research and Development, vol. 45, 2001.

[11] D. Cutting, J. Kupiec, J. Pedersen, and P. Sibun, "A practical part-of-speech tagger," in Proc. Third Conf. Applied Natural Language Processing, 1992, pp. 133-140.

[12] D. M. Bikel, R. Schwartz, and R. M. Weischedel, "An algorithm that learns what's in a name," Machine Learning, vol. 34, pp. 211-231, Feb. 1999.

[13] A. Dempster, N. Laird, and D. Rubin, "Maximum likelihood from incomplete data via the EM algorithm," Journal of the Royal Statistical Society, vol. 39, pp. 1-38, 1977.

[14] A. Corduneanu and T. Jaakkola, "Continuation methods for mixing heterogeneous sources," in Proc. of the 18th Annual Conference on Uncertainty in Artificial Intelligence (UAI), 2002.

[15] S. N. Chow, J. Mallet-Paret, and J. A. Yorke, "Finding zeros of maps: homotopy methods that are constructive with probability one," Math. Comput., vol. 32, pp. 887-899, 1978.

[16] L. T. Watson, M. Sosonkina, R. C. Melville, A. P. Morgan, and H. F. Walker, "Algorithm 777: HOMPACK90: A suite of Fortran 90 codes for globally convergent homotopy algorithms," ACM Trans. Math. Software, vol. 23, pp. 514-549, 1997.

[17] E. L. Allgower and K. Georg, Numerical Continuation Methods: an Introduction. New York: Springer-Verlag, 1990.

[18] T. M. Cover and J. A. Thomas, Elements of Information Theory. New York, NY: Wiley, 1991.

[19] R. M. Gray, "Vector quantization,” IEEE ASSP Magazine, pp. 4-29, Apr. 1984. 


\section{APPENDIX I}

\section{ProOf OF TheOREM 2}

Proof: First, since $B$ is topologically equivalent to the closed unit ball, it suffices to consider $B=\left\{x \in \mathbb{R}^{n} \mid\|x\|_{2} \leq 1\right\}$. Because of the rank assumption on $D_{b} a(b, z), H(b, \lambda, z)$ is transversal to zero. Therefore, as in [15], by the Parameterized Sard's Theorem, $H_{b}(\lambda, z)$ is also transversal to zero for almost all $b \in U$. This fact, as shown in [15] or [16], implies the existence and nonintersection properties of $\gamma$. It also follows (again from [15] or [16]) that $\gamma$ cannot just stop or wander around forever in $(0,1) \times \operatorname{int} B$. Therefore $\gamma$ must either return to $\lambda=0$, penetrate the boundary of the cylinder $[0,1) \times B$ at $0<\lambda<1$, or reach a point $(1, \bar{z})$. $\gamma$ cannot return to $\lambda=0$ because $a_{b}(z)$ had a unique nonsingular fixed point $z_{b}$. Consider any point $(\hat{\lambda}, \hat{z})$ on the boundary of $[0,1) \times B$ where $0<\hat{\lambda}<1,\|\hat{z}\|_{2}=1$. Now (writing simply $x y$ for the inner product of $x, y \in \mathbb{R}^{n}$ )

$$
\hat{z} H_{b}(\hat{\lambda}, \hat{z})=(1-\hat{\lambda})\left(\hat{z} \hat{z}-\hat{z} a_{b}(\hat{z})\right)+\hat{\lambda}(\hat{z} \hat{z}-\hat{z} f(\hat{z}))>0
$$

since $\hat{z} \hat{z}=1,\left|\hat{z} a_{b}(\hat{z})\right| \leq\|\hat{z}\|_{2}\left\|a_{b}(\hat{z})\right\|_{2}<1$, and $|\hat{z} f(\hat{z})| \leq\|\hat{z}\|_{2}\|f(\hat{z})\|_{2} \leq 1$. Therefore

$H_{b}(\hat{\lambda}, \hat{z}) \neq 0$, and $\gamma$ cannot penetrate the boundary for $0<\hat{\lambda}<1$. All that remains is that $\gamma$ must reach a point $(1, \bar{z})$, at which $\bar{z}=f(\bar{z})$.

As in [15] or [16], the finite arc length of $\gamma$ follows the transversality of $H_{b}(\lambda, z)$ and the full rank of $I-D f(\bar{z})$.

\section{APPENDIX II}

\section{NEW HMM VARIABLES}

The basic variables in HMMs, such as $\gamma_{t}(t)$ and $\xi_{t}(i, j)$ [8], are not amenable to computing the Jacobian matrices for the implementation of the homotopy method, . We therefore define a set of new HMM variables $(\psi, \phi$, and $\Phi)$ to facilitate this computation. They are defined as follows: for $t=1, \cdots, T$ and $t^{\prime}=1, \cdots, t-1$,

$$
\begin{aligned}
& \psi_{t, t^{\prime}}^{L}(i, j)=p\left(o_{t^{\prime}+1}, \cdots, o_{t-1}, s_{t}=i \mid s_{t^{\prime}}=j\right) \\
& \phi_{t, t^{\prime}}^{L}(i, j)=p\left(s_{t}=i \mid o_{t^{\prime}+1}, \cdots, o_{T}, s_{t^{\prime}}=j\right)
\end{aligned}
$$


for $t=1, \cdots, T-1$ and $t^{\prime}=1, \cdots, t$

$$
\Phi_{t, t^{\prime}}^{L}(i, j, k)=p\left(s_{t}=i, s_{t+1}=j \mid o_{t^{\prime}+1}, \cdots, o_{T}, s_{t^{\prime}}=k\right),
$$

where the superscript $L$ indicates that $t^{\prime} \leq t$.

Similarly, for $t=1, \cdots, T$ and $t^{\prime}=t+1, \cdots, T$,

$$
\begin{aligned}
& \psi_{t, t^{\prime}}^{R}(i, j)=p\left(o_{t+1}, \cdots, o_{t^{\prime}-1}, s_{t^{\prime}}=j \mid s_{t}=i\right)=\psi_{t^{\prime}, t}^{L}(j, i), \\
& \phi_{t, t^{\prime}}^{R}(i, j)=p\left(s_{t}=i \mid o_{1}, \cdots, o_{t^{\prime}-1}, s_{t^{\prime}}=j\right)
\end{aligned}
$$

for $t=1, \cdots, T-1$ and $t^{\prime}=t+1, \cdots, T$,

$$
\Phi_{t, t^{\prime}}^{R}(i, j, k)=p\left(s_{t}=i, s_{t+1}=j \mid o_{1}, \cdots, o_{t^{\prime}-1}, s_{t^{\prime}}=k\right),
$$

where the superscript $R$ indicates $t^{\prime} \geq t$.

The computation of $\psi$ is initialized at $t^{\prime}=t$ with

$$
\phi_{t, t}(i, j)=\frac{\delta(i, j)}{b_{i}\left(o_{t}\right)},
$$

and we can compute the rest of the $\psi$ iteratively as follows:

$$
\begin{aligned}
\psi_{t, t^{\prime}-1}^{L}(i, j) & =\sum_{k} \psi_{t, t^{\prime}}^{L}(i, k) a_{j k} b_{k}\left(o_{t^{\prime}}\right), \\
\psi_{t, t^{\prime}+1}^{R}(i, j) & =\sum_{k} \psi_{t, t^{\prime}}^{R}(i, k) a_{k j} b_{k}\left(o_{t^{\prime}}\right) .
\end{aligned}
$$

Finally, $\phi$ and $\Phi$ can be calculated based on $\psi$ as follows:

$$
\begin{aligned}
& \phi_{t, t^{\prime}}^{L}(i, j)=\psi_{t, t^{\prime}}(i, j) b_{i}\left(o_{t}\right) \frac{\beta_{t}(i)}{\beta_{t^{\prime}}(j)}, \\
& \phi_{t, t^{\prime}}^{R}(i, j)=\psi_{t, t^{\prime}}(i, j) b_{j}\left(o_{t^{\prime}}\right) \frac{\alpha_{t}(i)}{\alpha_{t^{\prime}}(j)}, \\
& \Phi_{t, t^{\prime}}^{L}(i, j, k)=\phi_{t+1, t}^{L}(j, i) \phi_{t, t^{\prime}}^{L}(i, k), \\
& \Phi_{t, t^{\prime}}^{R}(i, j, k)=\phi_{t, t+1}^{R}(i, j) \phi_{t+1, t^{\prime}}^{R}(j, k),
\end{aligned}
$$

where $\alpha_{t}(i)$ and $\beta_{t}(i)$ are defined the same as in [8], and they can be calculated efficiently via the standard forward-backward algorithm.

After computing of all the values of $\psi, \phi$, and $\Phi$, we remove the superscript $L$ and $R$ on them since the subscripts $t$ and $t^{\prime}$ have already encoded this information. 


\section{APPENDIX III}

\section{Computation of The JACOBIAN MATRICES}

To compute the Jacobian matrices $\nabla_{\tilde{\Theta}} E M_{0}(\Theta)$ and $\nabla_{\tilde{\Theta}} E M_{1}(\Theta)$, we need to compute the gradients of $p(y \mid \boldsymbol{x}, \Theta), \gamma_{t}(i)$ and $\xi_{t}(i, j)$ with respect to the parameters of the HMM classifier $\tilde{\Theta}$. This in turn requires the partial derivatives of $\log p(\boldsymbol{x} \mid \theta), \gamma_{t}(i)$ and $\xi_{t}(i, j)$ with respect to each element of $\theta=\left\{\pi^{N}, A^{N \times N}, B^{N \times M}\right\}$. Their computational formulas are specified in detail as follows:

$$
\begin{aligned}
& \frac{\partial \log p(\boldsymbol{x} \mid \theta)}{\partial \pi_{i}}=\frac{1}{p(\boldsymbol{x} \mid \theta)} \cdot \sum_{s_{1}, \cdots, s_{T}}\left[\frac{\partial \pi_{s_{1}}}{\partial \pi_{i}} \prod_{t=1}^{T-1} a_{s_{t} s_{t+1}} \prod_{t=1}^{T} b_{s_{t}}\left(x_{t}\right)\right] \\
& =\frac{1}{p(\boldsymbol{x} \mid \theta)} \cdot \sum_{s_{1}, \cdots, s_{T}}\left[\delta\left(s_{1}=i\right) \prod_{t=1}^{T-1} a_{s_{t} s_{t+1}} \prod_{t=1}^{T} b_{s_{t}}\left(x_{t}\right)\right] \\
& =\frac{1}{p(\boldsymbol{x} \mid \theta)} \cdot \sum_{s_{1}} \delta\left(s_{1}=i\right) b_{s_{1}}\left(x_{1}\right) \sum_{s_{2}, \cdots, s_{T}}\left[\prod_{t=1}^{T-1} a_{s_{t} s_{t+1}} \prod_{t=2}^{T} b_{s_{t}}\left(x_{t}\right)\right] \\
& =\frac{1}{p(\boldsymbol{x} \mid \theta)} \cdot b_{i}\left(x_{1}\right) \beta_{1}(i)=\gamma_{1}(i) / \pi_{i} \\
& \frac{\partial \log p(\boldsymbol{x} \mid \theta)}{\partial a_{i j}}=\frac{1}{p(\boldsymbol{x} \mid \theta)} \cdot \sum_{s_{1}, \cdots, s_{T}}\left[\pi_{s_{1}} \prod_{t=1}^{T} b_{s_{t}}\left(x_{t}\right) \frac{\partial}{\partial a_{i j}} \prod_{t=1}^{T-1} a_{s_{t} s_{t+1}}\right] \\
& =\frac{1}{p(\boldsymbol{x} \mid \theta)} \cdot \sum_{s_{1}, \cdots, s_{T}}\left[\pi_{s_{1}} \prod_{t=1}^{T} b_{s_{t}}\left(x_{t}\right) \sum_{k=1}^{T-1}\left(\delta\left(s_{k}=i, s_{k+1}=j\right) \prod_{t=1, t \neq k}^{T-1} a_{s_{t} s_{t+1}}\right)\right] \\
& =\frac{1}{p(\boldsymbol{x} \mid \theta)} \cdot \sum_{k=1}^{T-1}\left[\sum_{s_{1}, \cdots, s_{T}}\left(\pi_{s_{1}} \prod_{t=1}^{T} b_{s_{t}}\left(x_{t}\right) \delta\left(s_{k}=i, s_{k+1}=j\right) \prod_{t=1, t \neq k}^{T-1} a_{s_{t} s_{t+1}}\right)\right] \\
& =\frac{1}{p(\boldsymbol{x} \mid \theta)} \cdot \sum_{t=1}^{T-1} \alpha_{t}(i) b_{j}\left(x_{t+1}\right) \beta_{t+1}(j)=\frac{\sum_{t=1}^{T-1} \xi_{t}(i, j)}{a_{i j}} \\
& \frac{\partial \log p(\boldsymbol{x} \mid \theta)}{\partial b_{i}(v)}=\frac{1}{p(\boldsymbol{x} \mid \theta)} \cdot \sum_{t=1}^{T} \frac{\alpha_{t}(i) \beta_{t}(i)}{b_{i}\left(x_{t}\right)} \delta\left(x_{t}=v\right)=\frac{\sum_{t=1}^{T} \gamma_{t}(i) \delta\left(x_{t}=v\right)}{b_{i}(v)}
\end{aligned}
$$

where $\gamma_{t}(i)$ and $\xi_{t}(i, j)$ are defined the same as in [8], and they can be calculated efficiently from $\alpha_{t}(i)$ and $\beta_{t}(i)$. 
Similarly, the partial derivatives of $\gamma_{t}(i)$ and $\xi_{t}(i, j)$ can be calculated as

$$
\begin{aligned}
& \frac{\partial \gamma_{t}(i)}{\partial \pi_{k}}=\frac{\gamma_{t}(i)}{\pi_{k}}\left[\phi_{1, t}(k, i)-\gamma_{1}(k)\right] \\
& \frac{\partial \gamma_{t}(i)}{\partial a_{k l}}=\frac{\gamma_{t}(i)}{a_{k l}}\left[\sum_{t^{\prime}=1}^{T-1} \Phi_{t^{\prime}, t}(k, l, i)-\sum_{t^{\prime}=1}^{T-1} \xi_{t^{\prime}}(k, l)\right] \\
& \frac{\partial \gamma_{t}(i)}{\partial b_{k}(v)}=\frac{\gamma_{t}(i)}{b_{k}(v)}\left[\sum_{t^{\prime}=1}^{T}\left(\phi_{t^{\prime}, t}(k, i)-\gamma_{t^{\prime}}(k)\right) \delta\left(o_{t^{\prime}}=v\right)\right] \\
& \frac{\partial \xi_{t}(i, j)}{\partial \pi_{k}}=\frac{\xi_{t}(i, j)}{\pi_{k}}\left[\phi_{1, t}(k, i)-\gamma_{1}(k)\right] \\
& \frac{\partial \xi_{t}(i, j)}{\partial a_{k l}}=\frac{\xi_{t}(i, j)}{a_{k l}}\left[\sum_{t^{\prime}=1}^{t-1} \Phi_{t^{\prime}, t}(k, l, i)+\delta(k=i, l=j)+\sum_{t^{\prime}=t+1}^{T-1} \Phi_{t^{\prime}, t+1}(k, l, j)\right. \\
& \left.\quad-\sum_{t^{\prime}=1}^{T-1} \xi_{t^{\prime}}(k, l)\right] \\
& \frac{\partial \xi_{t}(i, j)}{\partial b_{k}(v)}=\frac{\xi_{t}(i, j)}{b_{k}(v)}\left[\sum_{t^{\prime}=1}^{t} \phi_{t^{\prime}, t}(k, i) \delta\left(o_{t^{\prime}}=v\right)+\sum_{t^{\prime}=t+1}^{T} \phi_{t^{\prime}, t+1}(k, j) \delta\left(o_{t^{\prime}}=v\right)\right. \\
& \left.\quad-\sum_{t^{\prime}=1}^{T} \gamma_{t^{\prime}}(k) \delta\left(o_{t^{\prime}}=v\right)\right]
\end{aligned}
$$

By the chain rule, the partial derivatives with respect to each element of $\tilde{\Theta}$ can be calculated as, for example

$$
\frac{\partial \gamma_{t}(i)}{\partial \tilde{\pi}_{k}}=\sum_{j} \frac{\partial \gamma_{t}(i)}{\partial \pi_{j}} \cdot \frac{\partial \pi_{j}}{\partial \tilde{\pi}_{k}}=\left(\frac{\partial \gamma_{t}(i)}{\partial \pi_{k}}-\sum_{j} \pi_{j} \frac{\partial \gamma_{t}(i)}{\partial \pi_{j}}\right) / \sum_{j} \tilde{\pi}_{j}
$$

and similarly for $\tilde{a}_{k l}$ and $\tilde{b}_{k}(v)$.

Finally, the partial derivatives of $p(y \mid \boldsymbol{x}, \Theta)$ with respect to each element of $\tilde{\Theta}$ are given as follows:

$$
\begin{aligned}
& \frac{\partial p(y \mid \boldsymbol{x}, \Theta)}{\partial \tilde{w}^{y^{\prime}}}=\varepsilon / \tilde{w}^{y^{\prime}} \\
& \frac{\partial p(y \mid \boldsymbol{x}, \Theta)}{\partial \tilde{\pi}_{k}^{y^{\prime}}}=\varepsilon / \tilde{\pi}_{k}^{y^{\prime}} \cdot\left[\gamma_{1}^{y^{\prime}}(k)-\pi_{k}^{y^{\prime}}\right] \\
& \frac{\partial p(y \mid \boldsymbol{x}, \Theta)}{\partial \tilde{a}_{k l}^{y^{\prime}}}=\varepsilon / \tilde{a}_{k l}^{y^{\prime}} \cdot\left[\sum_{t=1}^{T-1} \xi_{t}^{y^{\prime}}(k, l)-a_{k l}^{y^{\prime}} \sum_{t=1}^{T-1} \gamma_{t}^{y^{\prime}}(k)\right]
\end{aligned}
$$




$$
\frac{\partial p(y \mid \boldsymbol{x}, \Theta)}{\partial \tilde{b}_{k}^{y^{\prime}}(v)}=\varepsilon / \tilde{b}_{k}^{y^{\prime}}(v) \cdot\left[\sum_{t=1}^{T} \gamma_{t}^{y^{\prime}}(k) \delta\left(o_{t}=v\right)-b_{k}^{y^{\prime}}(v) \sum_{t=1}^{T} \gamma_{t}^{y^{\prime}}(k)\right]
$$

where

$$
\varepsilon=p\left(y^{\prime} \mid \boldsymbol{x}, \Theta\right) \cdot\left[\delta\left(y=y^{\prime}\right)-p(y \mid \boldsymbol{x}, \Theta)\right]
$$

